\title{
On the holistic validation of electronic materials compound for irradiation study - Experimental and calculated results
}

\author{
Thomas Frosio $^{1}$ D $\cdot$ Nabil Menaa ${ }^{1} \cdot$ Matteo Magistris $^{1} \cdot$ Chris $^{\text {Theis }}{ }^{1}$
}

Received: 8 June 2020 / Published online: 12 August 2020

(c) The Author(s) 2020

\begin{abstract}
Due to the large variations of chemical compositions in electronic material, the estimation of the radionuclide inventory following irradiation represents a technical challenge at CERN high-energy particle accelerators. In particular, the activation of printed circuit boards is of concern to the CERN experiments as they are widely used for various purposes ranging from safety systems to sub-detector controls. Because of maintenance operations, part of this equipment has to be removed from the accelerator machines. The literature provides a variety of compositions for electronic materials, leaving the problematic selection of the most appropriate composition for an activation study to the reader. In this article, we discuss two reference chemical compositions on the basis of a statistical analysis of large datasets of gamma spectroscopy results, and on ActiWiz calculations which take into account different activation scenarios at CERN. These results can be extended to electronic material irradiated in other particle accelerators.
\end{abstract}

Keywords Nuclide inventories $\cdot$ Electronic components $\cdot$ ActiWiz Activation calculation

\section{Introduction}

Operating a high-energy proton accelerator like the Large Hadron Collider (LHC) presents numerous challenges with respect to operational radiation protection and the assessment of possible radiation risks. The activation of material is of great interest both in view of maintenance and for waste disposal at the end of its life cycle. In particular, the activation of printed circuit boards (PCB) is of concern to the CERN experiments as they are widely used for various purposes ranging from safety systems to sub-detector controls. Because of maintenance operations, part of this equipment has to be removed from the accelerator machines. It is then temporarily stored awaiting radiological classification, followed by repair and reuse, or by final disposal as waste. Both reuse and final disposal require knowledge of the radionuclide inventory of such material or at least a reasonably penalizing radionuclide inventory.

The purpose of this work is to provide a consistent and viable chemical composition to perform numerical

Thomas Frosio

thomas.frosio@gmail.com

1 Radiation Protection Group, European Organization for Nuclear Research, 1211 Geneva 23, Switzerland simulation studies that yields reasonable results. These simulations are of interest for predicting the radionuclide inventory during design, operations, and waste elimination phases. Numerical simulations depend on activation scenario and chemical compositions. Of course, the activation scenarios (primary particle energy, location in the accelerator, irradiation and cooling times) have a huge impact on the radionuclide inventory but they are generally well known or treated statistically in a way to construct a reasonably penalizing radionuclide inventory. Regarding chemical compositions, the wide range of chemical elements and associated mass fractions that are present in electronic components induce some difficulties to perform numerical simulations for risk assessment or waste characterization. Indeed, the large variety of chemical compositions induce a large variety of radionuclide inventories produced.

In order to be able to acquire the necessary data to propose a "standard" composition for radiation protection studies at CERN regarding electronic components, we perform, in this work, comparisons between numerical simulations and experimental measurements. Numerical simulations in the present study are based on ActiWiz [1] calculations and measurements come from gamma spectroscopy. ActiWiz is developed by CERN. It is a deterministic calculation code that provides radionuclide inventories and associated 
radiological hazards. It takes as inputs an irradiated material elemental composition, a radiation field (secondary particle fluences) and an irradiation and decay profile. The beam losses effects constitute the main cause of the activation in the CERN accelerator complex. As, the primary protons interact with the surrounding environment, they produce secondary hadrons due to inelastic collisions. The secondary particles that contribute to the activation are: neutrons, photons, pions as well as protons.

For the numerical simulations, we consider two kinds of material compositions encountered in the literature for electronic boards. In general, a very large variety of compositions is encountered, depending on the type of electronics and its use. This also holds true for PCBs found at CERN and therefore, the assumed compounds are based on averages that have been encountered in the waste stream [2,3]. Also, the whole set of activation scenarios that can occur at CERN are considered. Note that the analysis results are easily transposable to other accelerator facilities.
The results of the calculations are then compared to a database of 218 gamma spectroscopy measurements performed from 2004 to 2019 on PCBs irradiated at CERN's accelerator complex. The trends are qualitatively analyzed and conclusions are drawn to improve the knowledge on PCB material composition for a better consistency between the calculations and measurements.

\section{Description of the samples}

218 samples of electronic equipment are measured with high resolution HPGe gamma spectroscopy from 2004 to 2019. Examples of these samples are shown in Fig. 1. The counting time for most of the items was in the order of about $10,000 \mathrm{~s}$ (ranging from 1800 to $50,000 \mathrm{~s}$ depending on the contact dose rate and desired precision).

The gamma spectroscopy analyses include samples that originate from the whole CERN complex. Hence, the results remain comparable for a general / holistic comparison. In a
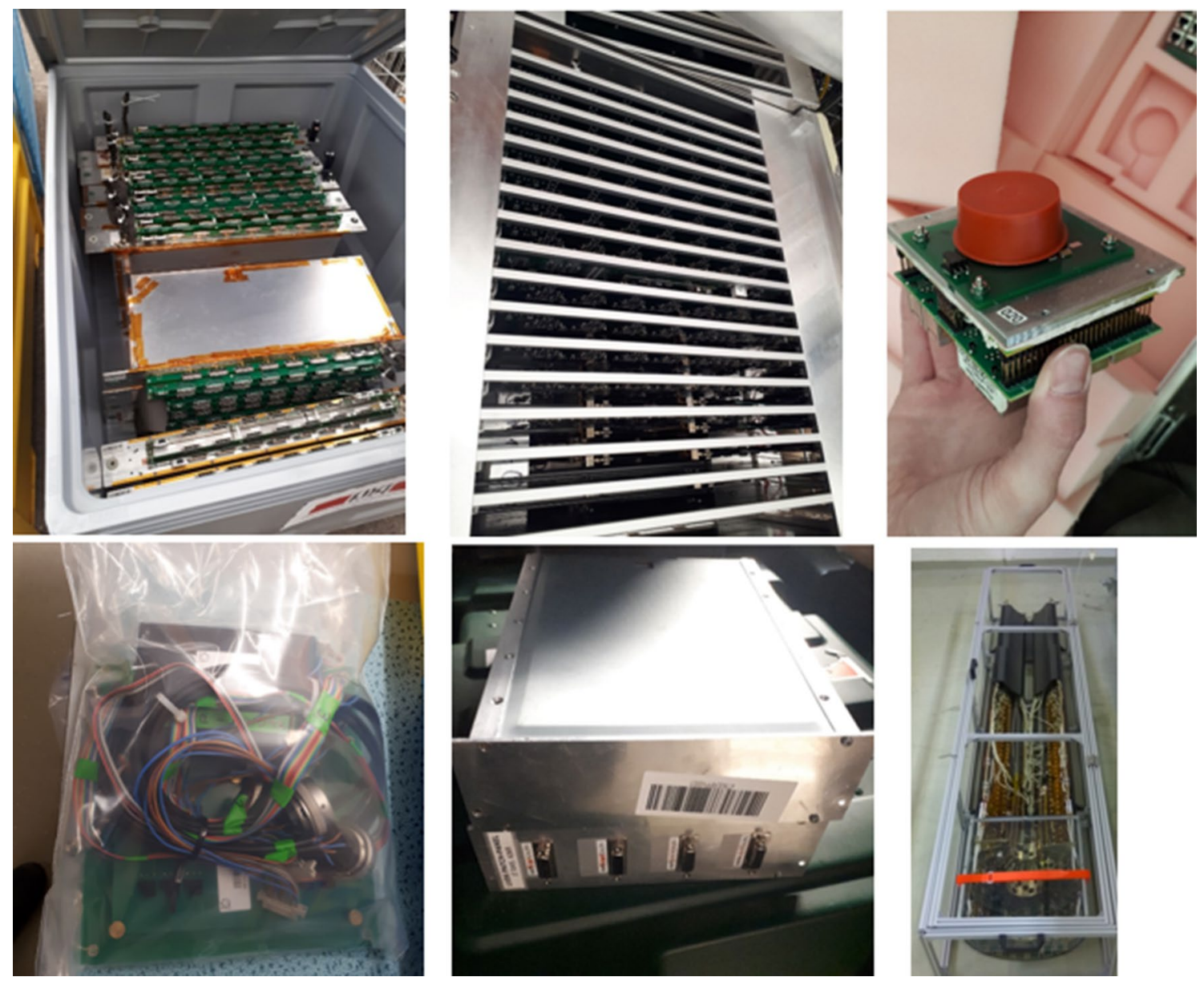

Fig. 1 Examples showing the diversity of electronic components. From top-left to bottom-right: Inner Tracker modules, front-end electronic modules, electronic boards from the Proton Synchrotron, electronic board with cables, radiation monitor system, detector electronics 
statistical point of view, the objective is to ensure that the numerical simulations do not provide high discrepancies compared to the experimental results, for the radionuclides that are of interest, i.e. radionuclides which radiological hazard are high. We show in this study that, for these radionuclides, the numerical simulations and experimental results are consistent.

\section{ActiWiz calculations}

Calculations are done with ActiWiz 3.3.15/2018 - 1010 [1] in order to compute the radionuclide inventory. Different scenarios are considered and associated fluences calculations are described in $[1,4]$ :

- All locations in the accelerator, from the beam line to behind the shielding,

- All machines energies from $160 \mathrm{MeV}$ to $7 \mathrm{TeV}$ and all large-scale LHC experiments (ATLAS, LHCb, CMS, ALICE) [4],

- Irradiation times from 1 day to 30 years,

- Cooling times from $3 \mathrm{~h}$ to 2 years in order to reflect the envelope conditions for the sample population that is measured.

- Two different material compositions are identified in the literature (see Refs. [2, 3]). They have been determined by studying electronics scrap that is mixed, shredded and analyzed to get an average composition.

13,000 calculations are carried out with the material compositions presented in Tables 1 and 2.

\section{Gamma spectroscopy setup}

Gamma spectroscopy analyses are carried out on 218 electronic parts sampled from material received between 2004 and 2019 using high purity germanium detectors. All the samples are irradiated at CERN's accelerator complex. ISOCS (In Situ Counting Object Software) and LabSOCS (Laboratory Sourceless Calibration Software) [5, 6] from Canberra are used in the laboratory for creating customized efficiency calibrations curves. The ISOCS/LabSOCS software overcomes the limitations of traditional (tedious and expensive) efficiency calibration techniques and allows for practical modelling and accurate assay of almost any object which need to be measured by gamma spectroscopy. With ISOCS/LabSOCS, each individual detector has a unique set of characteristics that are experimentally validated and used to generate the efficiency calibrations curves [7].

The geometry description can be visualized and edited in the ISOCS/LabSOCS Geometry Composer application which contains a visualization interface of the sample geometry. For these samples measurements, efficiency calibration

Table 1 Weight fractions for Goosey's material composition

\begin{tabular}{lllllllll}
\hline Goosey & Element & $\mathrm{Al}$ & $\mathrm{C}$ & $\mathrm{Cu}$ & $\mathrm{Au}$ & $\mathrm{H}$ & $\mathrm{Fe}$ & $\mathrm{Pb}$ \\
& Mass ratio & 0.05 & 0.51 & 0.16 & $2.50 \mathrm{E}-04$ & 0.05 & 0.05 & 0.02 \\
& Element & $\mathrm{Ni}$ & $\mathrm{O}$ & $\mathrm{Pd}$ & $\mathrm{Ag}$ & $\mathrm{Sn}$ & $\mathrm{Zn}$ & $\mathrm{Density}$ \\
& Mass ratio & 0.01 & 0.11 & $9.88 \mathrm{E}-05$ & $1.00 \mathrm{E}-03$ & 0.03 & 0.01 & $3.37 \mathrm{~g} / \mathrm{cm}^{3}$ \\
\hline
\end{tabular}

Table 2 Weight fractions for Kindesjo's material composition

\begin{tabular}{lllllllll}
\hline Kindesjo & Element & $\mathrm{Al}$ & $\mathrm{Sb}$ & $\mathrm{As}$ & $\mathrm{Ba}$ & $\mathrm{Be}$ & $\mathrm{Bi}$ & $\mathrm{Br}$ \\
& Mass ratio & 0.05 & $6.04 \mathrm{E}-04$ & $9.99 \mathrm{E}-05$ & $2.00 \mathrm{E}-03$ & $1.10 \mathrm{E}-05$ & $1.70 \mathrm{E}-03$ & 0.01 \\
& Element & $\mathrm{Cd}$ & $\mathrm{C}$ & $\mathrm{Cl}$ & $\mathrm{Cr}$ & $\mathrm{Cu}$ & $\mathrm{F}$ & $\mathrm{Ga}$ \\
& Mass ratio & $1.50 \mathrm{E}-04$ & 0.41 & 0.02 & $5.01 \mathrm{E}-04$ & 0.27 & $9.41 \mathrm{E}-04$ & $3.50 \mathrm{E}-04$ \\
& Element & $\mathrm{Au}$ & $\mathrm{H}$ & $\mathrm{I}$ & $\mathrm{Fe}$ & $\mathrm{Kr}$ & $\mathrm{Pb}$ & $\mathrm{Mn}$ \\
& Mass ratio & $8.01 \mathrm{E}-04$ & 0.03 & $2.00 \mathrm{E}-03$ & 0.05 & $1.00 \mathrm{E}-04$ & 0.02 & $4.70 \mathrm{E}-03$ \\
& Element & $\mathrm{Hg}$ & $\mathrm{Mo}$ & $\mathrm{Ni}$ & $\mathrm{O}$ & $\mathrm{Ag}$ & $\mathrm{S}$ & $\mathrm{Sn}$ \\
Mass ratio & $1.00 \mathrm{E}-05$ & $3.00 \mathrm{E}-05$ & $4.70 \mathrm{E}-03$ & 0.07 & $3.30 \mathrm{E}-03$ & $1.00 \mathrm{E}-03$ & 0.01 \\
& Element & $\mathrm{Ti}$ & $\mathrm{Zn}$ & $\mathrm{Zr}$ & & & $\mathrm{Density}$ \\
\\
Mass ratio & 0.03 & 0.02 & $3.00 \mathrm{E}-04$ & & & & $4.31 \mathrm{~g} / \mathrm{cm}^{3}$ \\
\hline
\end{tabular}


curves are produced considering an envelope geometry in order to slightly penalize, conservatively, the activity results, as discussed in [8].

\section{Comparison of predicted and measured radionuclides}

\section{Occurrence frequency of specific radioisotopes}

In order to validate the electronic material compositions used in this study, we consider a batch of 218 gamma spectroscopy analyses performed on activated electronic components at CERN. We consider a list of 98 radionuclides identified and quantified in the gamma spectroscopy results. Only radionuclides whose activities contribute by more than $1.0 \%$ of the total activity is considered in the radionuclide inventory of the ActiWiz calculations. Moreover, only radionuclides that appear in at least $5 \%$ of the gamma spectroscopy results (or ActiWiz calculations) are retained. This limits the number of radionuclides to compare to 27 instead of 98 .

Figure 2 provides a comparison of the frequencies of occurrence between experimental and calculation results for each radionuclide. We notice that the lists of radionuclides found in ActiWiz and gamma spectroscopy results are identical excluding few exceptional cases. The exceptions include natural radionuclides (such as K-40, Th-232, and $\mathrm{Ra}-226)$ and other radionuclides that are identified in gamma spectroscopy and not in ActiWiz calculations (such as Te123m, Sn-113, Ta-182, Rb-83, Se-75, Y-88) as it can be seen in Fig. 2. A discussion on the origin of these radionuclides is provided in "Investigation of radionuclides appearing in the measurements and not in the calculations" section.

Comparing the measured and calculated radionuclides, one needs to highlight the fact that the gamma spectroscopy results correspond to parts of electronic equipment that are not limited only to pure PCB boards. The equipment could be a mixture of electronic boards, cables, electronic modules often made of steel or aluminum and oscillators as it is illustrated in Fig. 1. Consequently, the measurements do not include only PCBs and this can naturally lead to some discrepancies with the ActiWiz calculations which are based on pure PCBs only. The presence of Cu-64 in the ActiWiz calculation can only be explained by the short half-life of $13 \mathrm{~h}$ and the inclusion of the rather short cooling time of $3 \mathrm{~h}$ in the set of simulation parameters. The $3 \mathrm{~h}$ cooling time is considered in order to allow investigating the radionuclides with very short half-lives. However, it is highly improbable that measurements were done so fast after removing the electronic parts from the accelerator complex. The reason for this difference between gamma spectroscopy results and calculations is the fact that gamma spectroscopy measurements are rarely performed any sooner than this time span of some hours.

All in all, the frequency of occurrence of the remaining radionuclides in ActiWiz matches the one in gamma spectroscopy measurements within a factor of 2 , and it is extremely similar in the case of V-48, Co-56 and Co-60. The existing discrepancies are mainly due to the following two reasons:

1 The detection limits of the gamma spectroscopy results have a direct impact on the frequency of occurrence. This explains the approximate factor of two between the gamma spectroscopy and ActiWiz calculations (see Fig. 2, e.g. Zn-65 found with a frequency of $80 \%$ in ActiWiz calculations and with a frequency of $40 \%$ in the measurements because of a high MDA).

2 The ActiWiz calculations are performed considering a uniform probability distribution of irradiation times, energies, locations, and cooling times with values below 2 years. On the other hand, the samples are selectively extracted from different locations at different accelerator machines energies. Hence, the probability distribution of the irradiation scenarios in the measurement is not uniform. This effect can be observed in particular with Be-7 for which the frequency of appearance is lower in the measurements compared to ActiWiz (see Fig. 2). For radionuclides with larger half-life, e.g. Co-60, measurements and calculation frequencies are consistent because they are less dependent on the large variety of time gaps between end of irradiation and measurement.

Another point to notice is that some radionuclides appear in measurements and ActiWiz calculations with Kindesjo's composition but not in ActiWiz calculations with Goosey's composition. These 5 radionuclides are $\mathrm{Sc}-46 / 47, \mathrm{Br}-82$ and $\mathrm{Sb}-122 / 124$ that are directly produced from elements titanium, bromine and antimony, included in Kindesjo's but not in Goosey's data.

In "Analysis of main radionuclides" section of this document, we focus on the 21 radionuclides, presented in Fig. 2, which appear in both measurements and calculations. Their corresponding activities are normalized to sum up to $100 \%$ of total activity. Then, in the following in "Investigation of radionuclides appearing in the measurements and not in the calculations" section, we consider the 6 radionuclides which appear only in measurements.

\section{Analysis of main radionuclides}

In order to validate the material compositions of Kindesjo and Goosey used in the ActiWiz calculations, comparisons of activity contributions (see Eq. 1) between measurements and ActiWiz are presented in Figs. 3, 4, 5, 6, 7, 8, 9, 10 and 

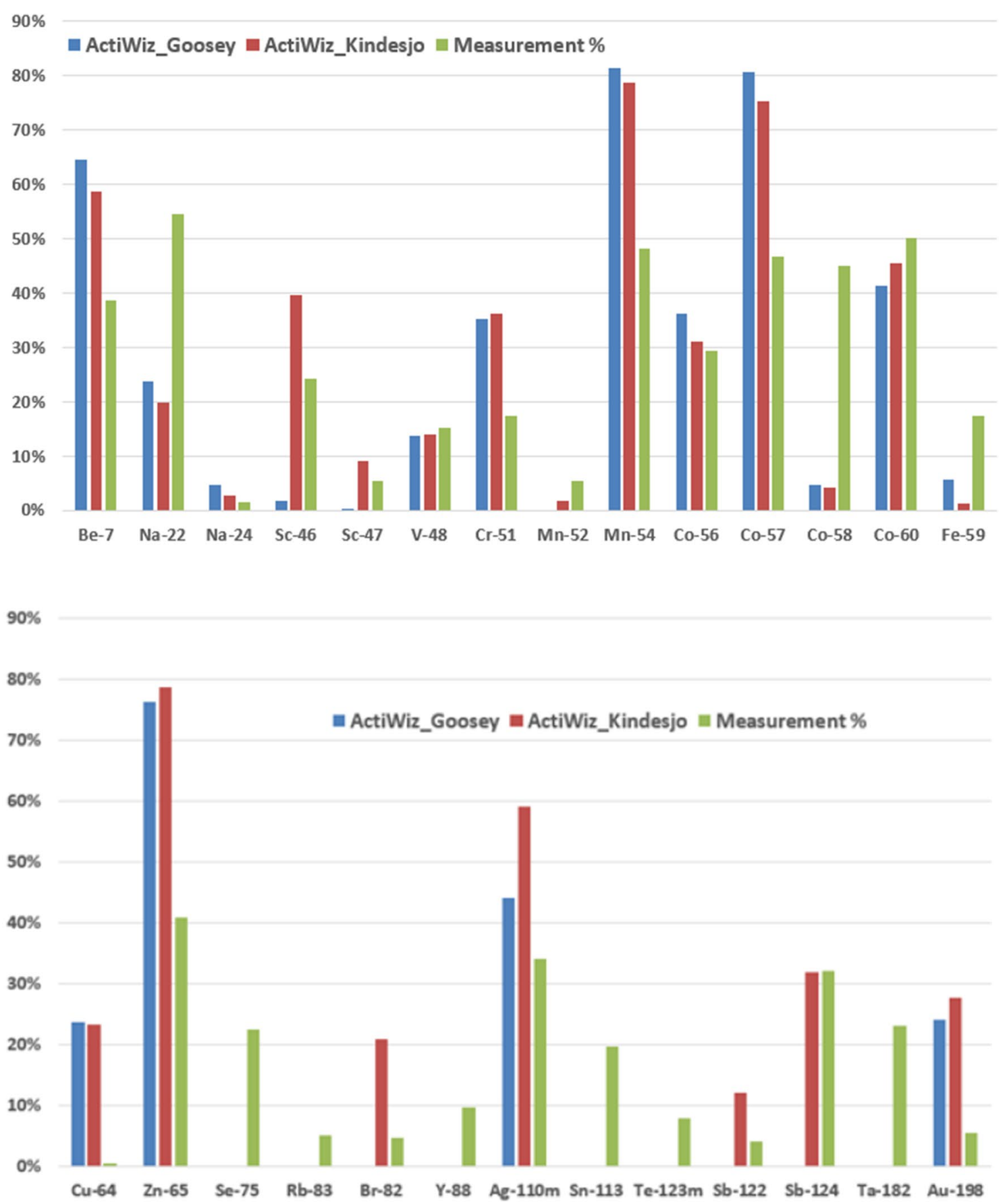

Fig. 2 Comparison of frequency of occurrence of radionuclides encountered in measurements and ActiWiz calculations of electronics

11 for some of the 28 main radionuclides for which a normalization is carried out. This normalization is performed in order to exclude the large set of radionuclides obtained by calculation with negligible activity values. The uncertainties presented in the next histograms are calculated as detailed in Eq. (2). We can see that for most of the cases (Figs. 3, 4, 5, $6,7)$, the activity contributions are similar. The medians, 1 st and 3rd quartiles are always within a factor of 2 . We mainly find discrepancies at high values of activity contributions above $40 \%$. As explained above, this effect is due to the non-identification of other radionuclides in the sample than the nuclides of interest (see Figs. 8, 9, 10, 11).

Equation 1 Activity contribution

$$
\text { Act.Contrib. }=\frac{a_{r n}}{\sum_{i} a_{i}}
$$




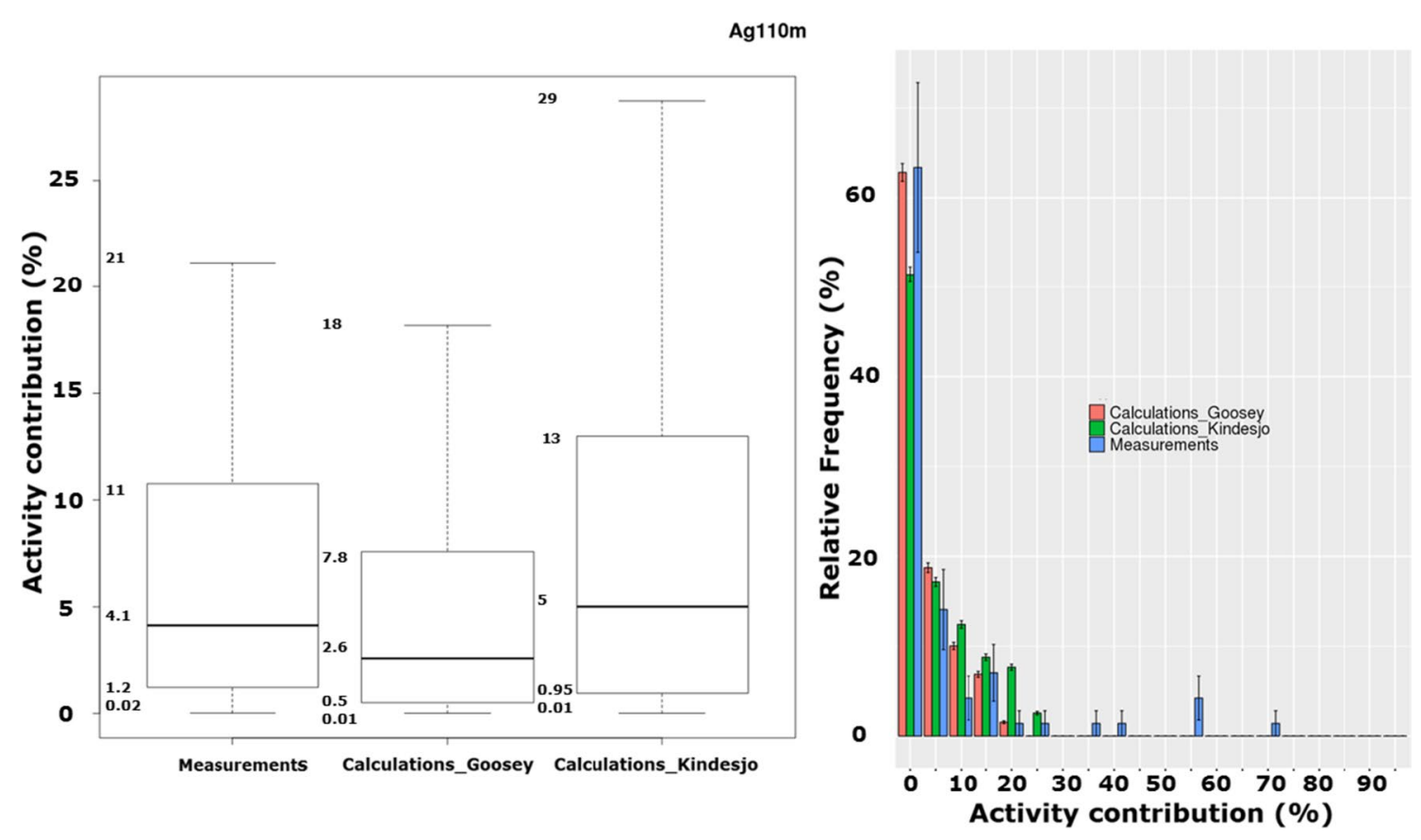

Fig. 3 Activity contribution comparison of measurements and ActiWiz calculations for Ag-110 m. The left figure represents a boxplot displaying first and third quartiles and the median (outliers excluded).
The right figure is a frequency histogram of the activity contributions. Uncertainties are presented at 1 sigma

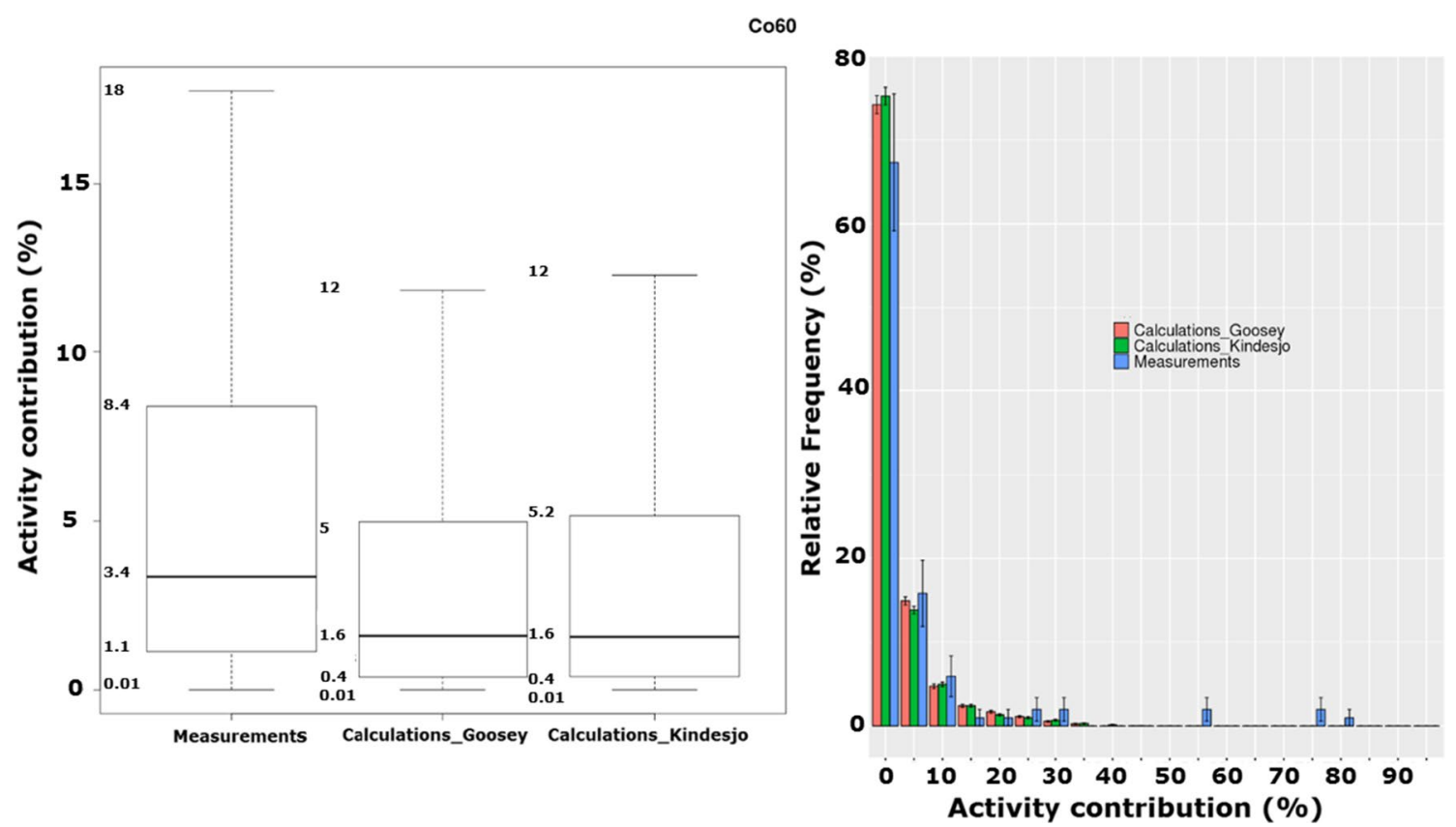

Fig. 4 Activity contribution comparison of measurements and ActiWiz calculations for Co-60. The left figure represents a boxplot displaying first and third quartiles and the median (outliers excluded).
The right figure is a frequency histogram of the activity contributions. Uncertainties are presented at 1 sigma 


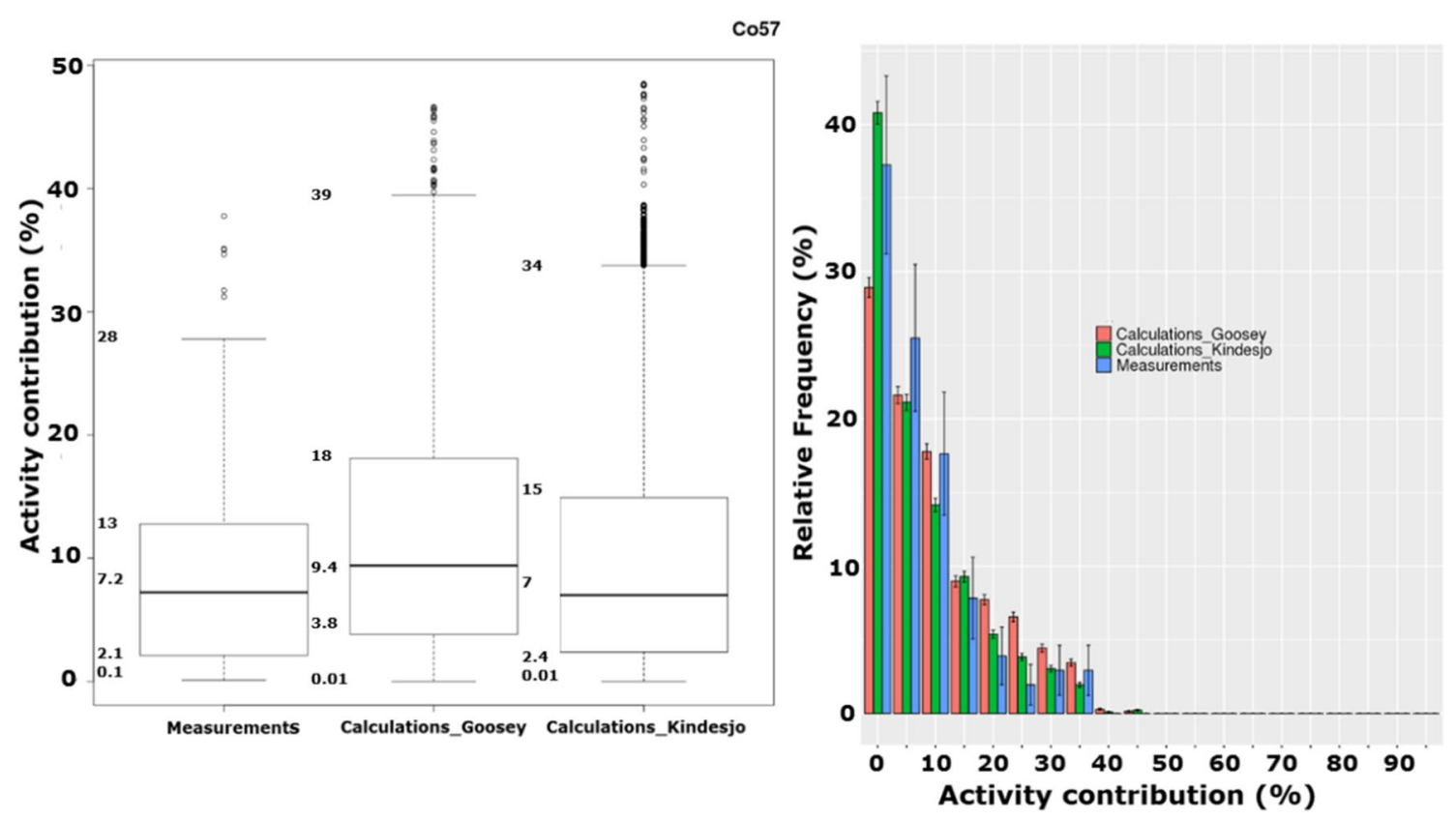

Fig. 5 Activity contribution comparison of measurements and ActiWiz calculations for Co-57. The left figure represents a boxplot displaying first and third quartiles and the median. The right figure is a frequency histogram of the activity contributions. Uncertainties are presented at 1 sigma

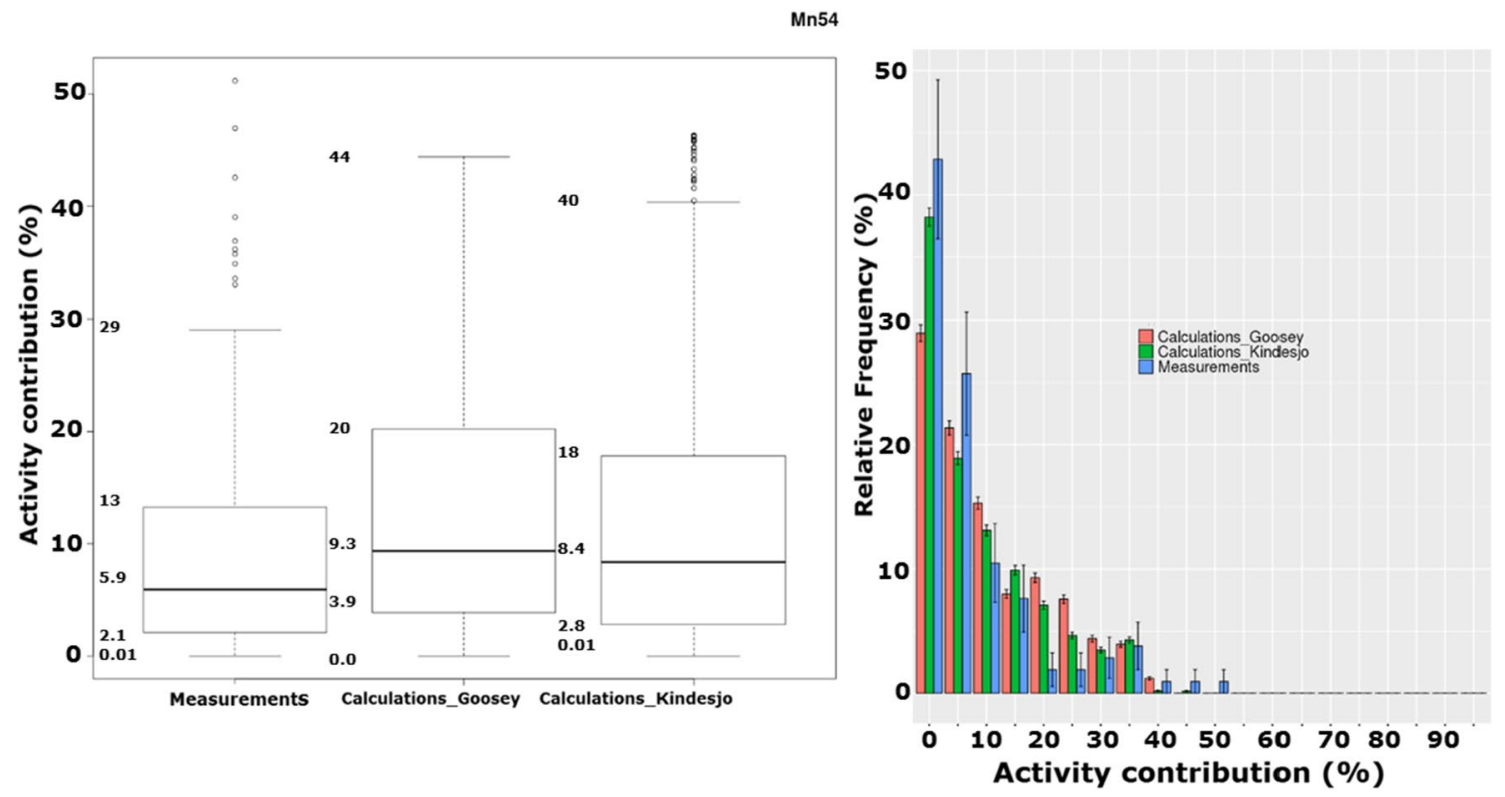

Fig. 6 Activity contribution comparison of measurements and ActiWiz calculations for Mn-54. The left figure represents a boxplot displaying first and third quartiles and the median. The right figure is

$a_{r n}$ is the activity measured (or calculated) for the radionuclide of interest in a specific irradiation scenario. $\sum_{i} a_{i}$ represents the sum of all the activities measured (or calculated) in a specific irradiation scenario.

Equation 2 Standard deviation of each bins frequency of histograms applying Poisson statistics to estimate count variation a frequency histogram of the activity contributions. Uncertainties are presented at 1 sigma

$\sigma\left(\frac{N_{i, i+1}}{N_{t}}\right) \approx \frac{\sqrt{N_{i, i+1}}}{N_{t}}$

$N_{i, i+1}$ is the count in the bin $\left[i, i+1\right.$ [ of the histogram. $N_{t}$ is the total count in the histogram. 


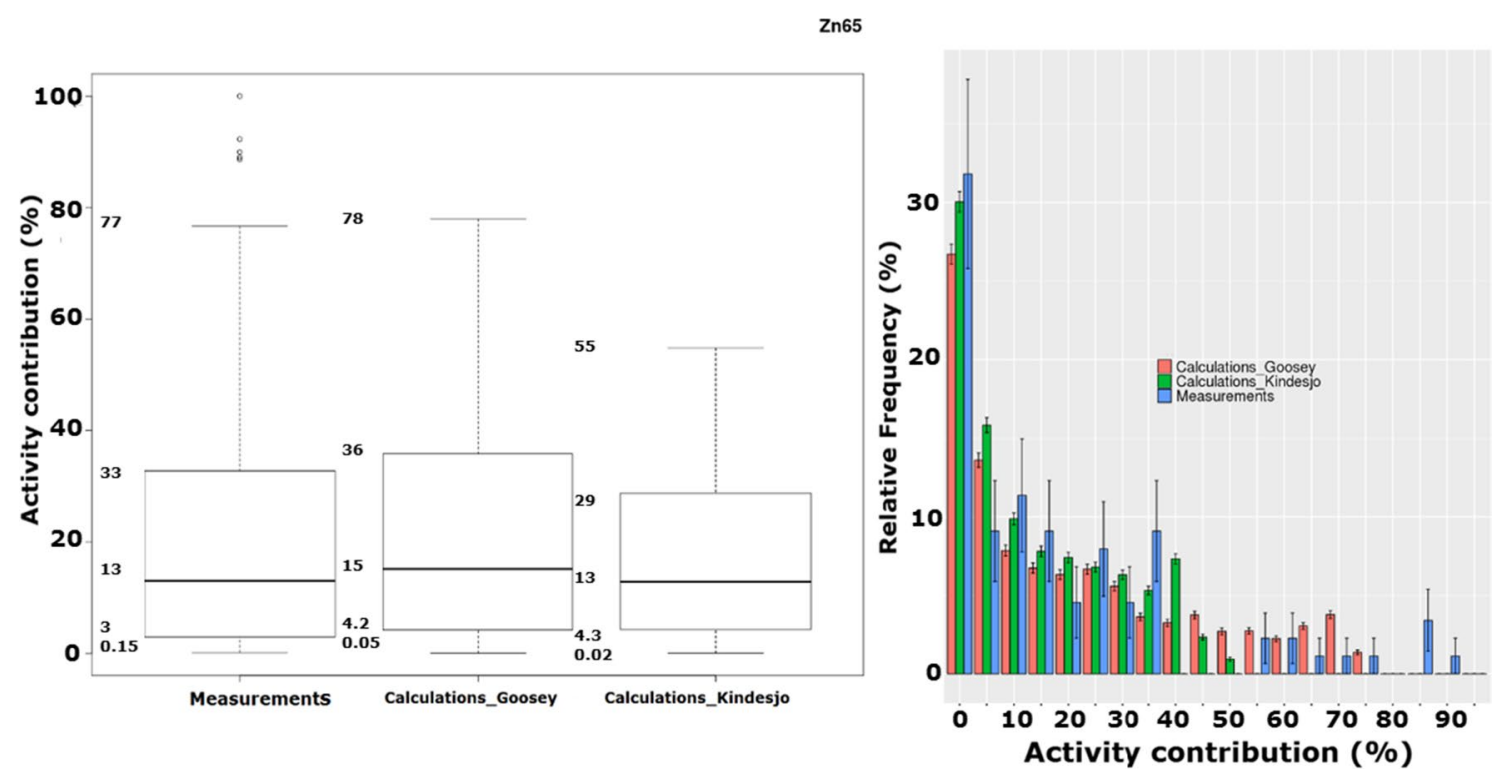

Fig. 7 Activity contribution comparison of measurements and ActiWiz calculations for $\mathrm{Zn}-65$. The left figure represents a boxplot displaying first and third quartiles and the median. The right figure is a frequency histogram of the activity contributions. Uncertainties are presented at 1 sigma

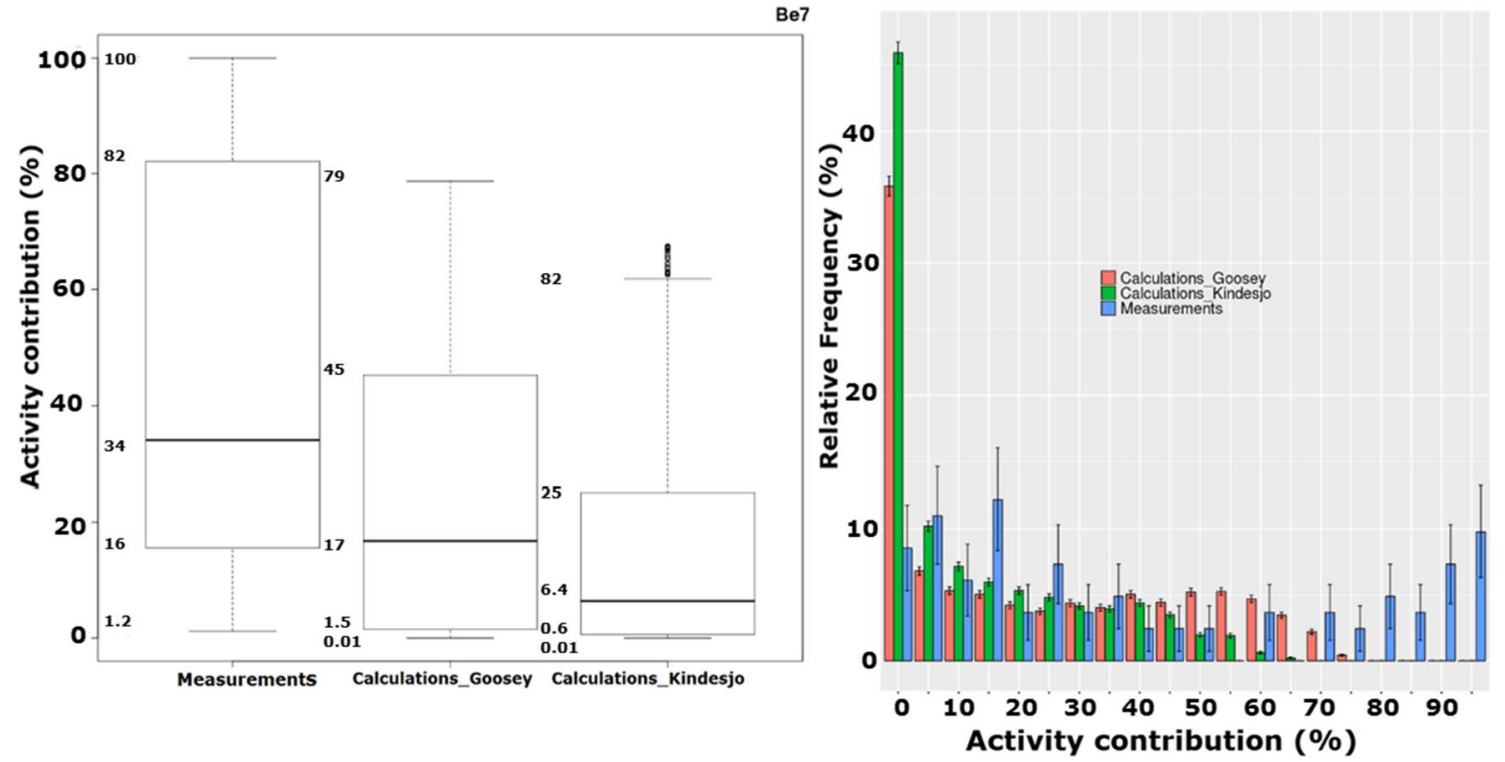

Fig. 8 Activity contribution comparison of measurements and ActiWiz calculations for Be-7. The left figure represents a boxplot displaying first and third quartiles and the median. The right figure is a frequency histogram of the activity contributions. Uncertainties are presented at 1 sigma

The Ag-110 m activity contributions are quite similar in both calculations and measurements. As illustrated in Fig. 3 (left boxplot), we find a median value at $3.5 \%$ with ActiWiz calculations and $4.5 \%$ with gamma spectroscopy results. Furthermore, the histograms in Fig. 3 are similar, with only few gamma spectroscopy results ranging from 40 to $100 \%$ of $\mathrm{Ag}-110 \mathrm{~m}$. Below 40\%, the distributions are in the range of the 2 sigma uncertainties due to the statistics (small number of measurements compared to calculations).

For Co-60, the activity contributions show higher discrepancies (compared to Ag-110 m), with a maximum value at $12 \%$ in the calculation and $18 \%$ in the measurements. The median is also lower in the case of the calculations compared to the measurements ( $1.6 \%$ compared to $3.4 \%$ ). This is also due to the fact that Co-60 is easy to measure, with low 


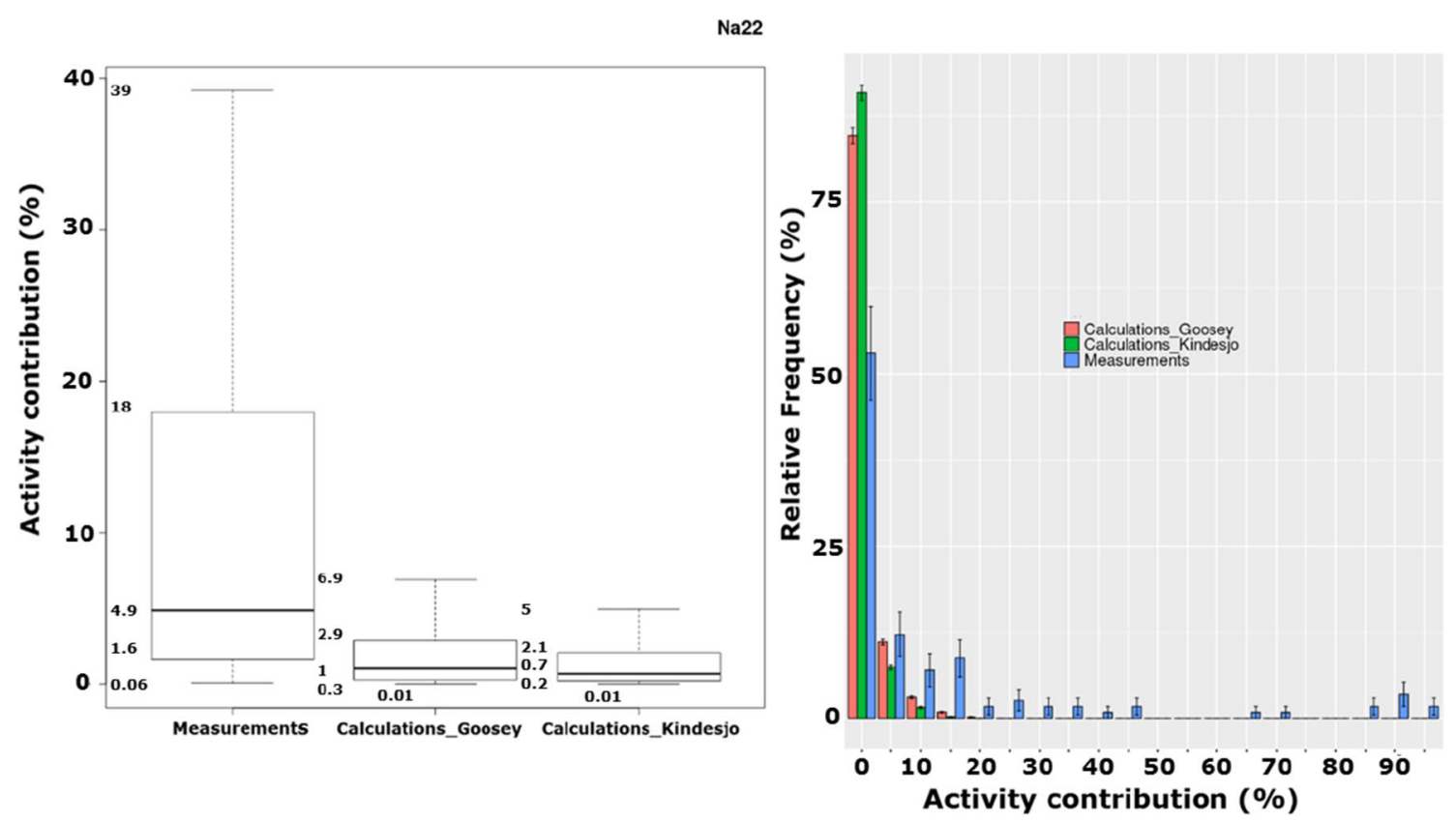

Fig. 9 Activity contribution comparison of measurements and ActiWiz calculations for Na-22. The left figure represents a boxplot displaying first and third quartiles and the median (outliers excluded).
The right figure is a frequency histogram of the activity contributions. Uncertainties are presented at 1 sigma

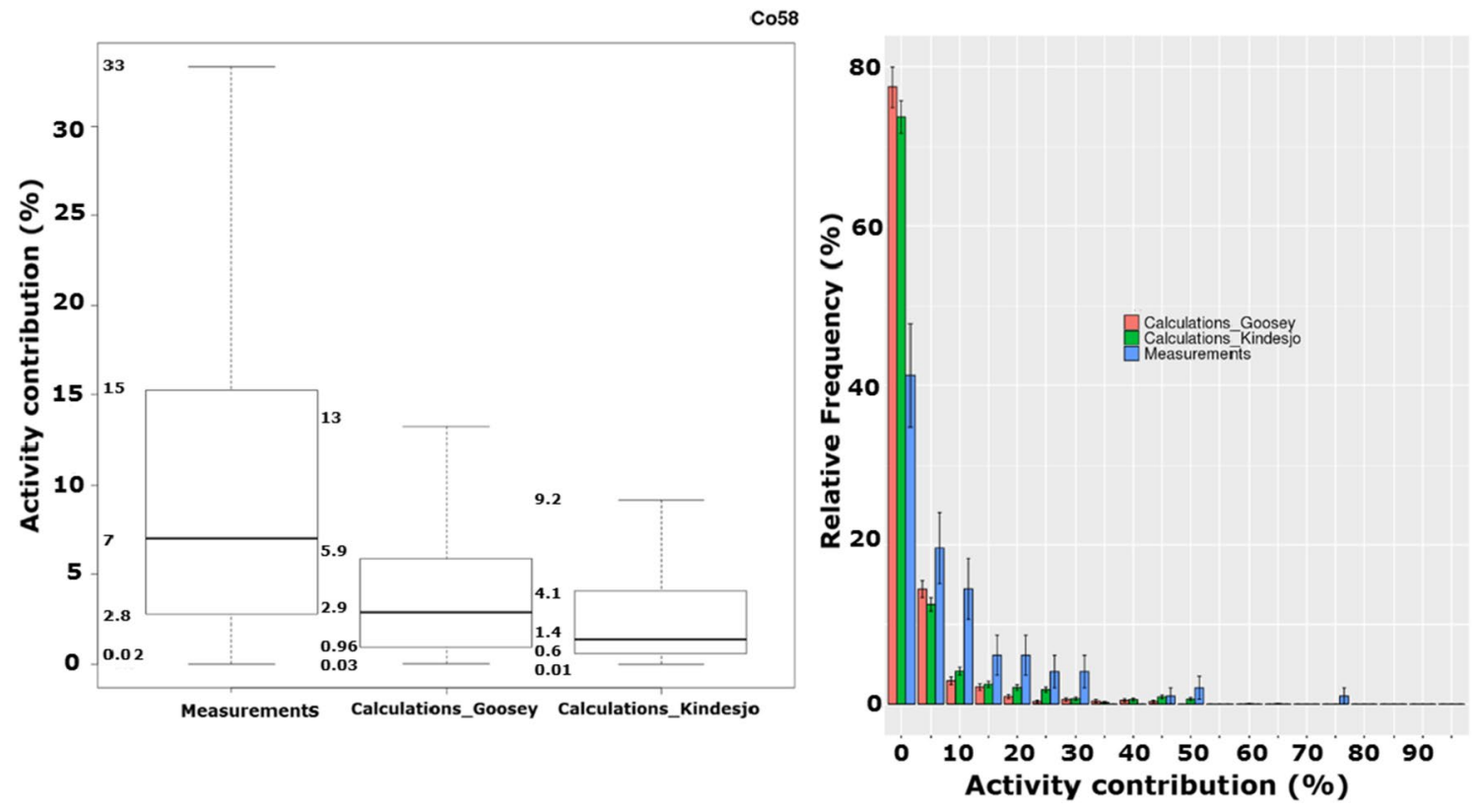

Fig. 10 Activity contribution comparison of measurements and ActiWiz calculations for Co-58. The left figure represents a boxplot displaying first and third quartiles and the median (outliers excluded).

MDA values. Consequently, it is identified more frequently in spectroscopy measurements than other radionuclides. Therefore, the activity contribution found in the spectroscopy results can be higher. However, we can conclude that
The right figure is a frequency histogram of the activity contributions. Uncertainties are presented at 1 sigma

the activity contributions shown in Fig. 4 are consistent between calculations and measurements.

Finally, the activity contributions for Co-57, Mn-54 and Zn-65 are similar as shown in Figs. 5, 6 and 7. 


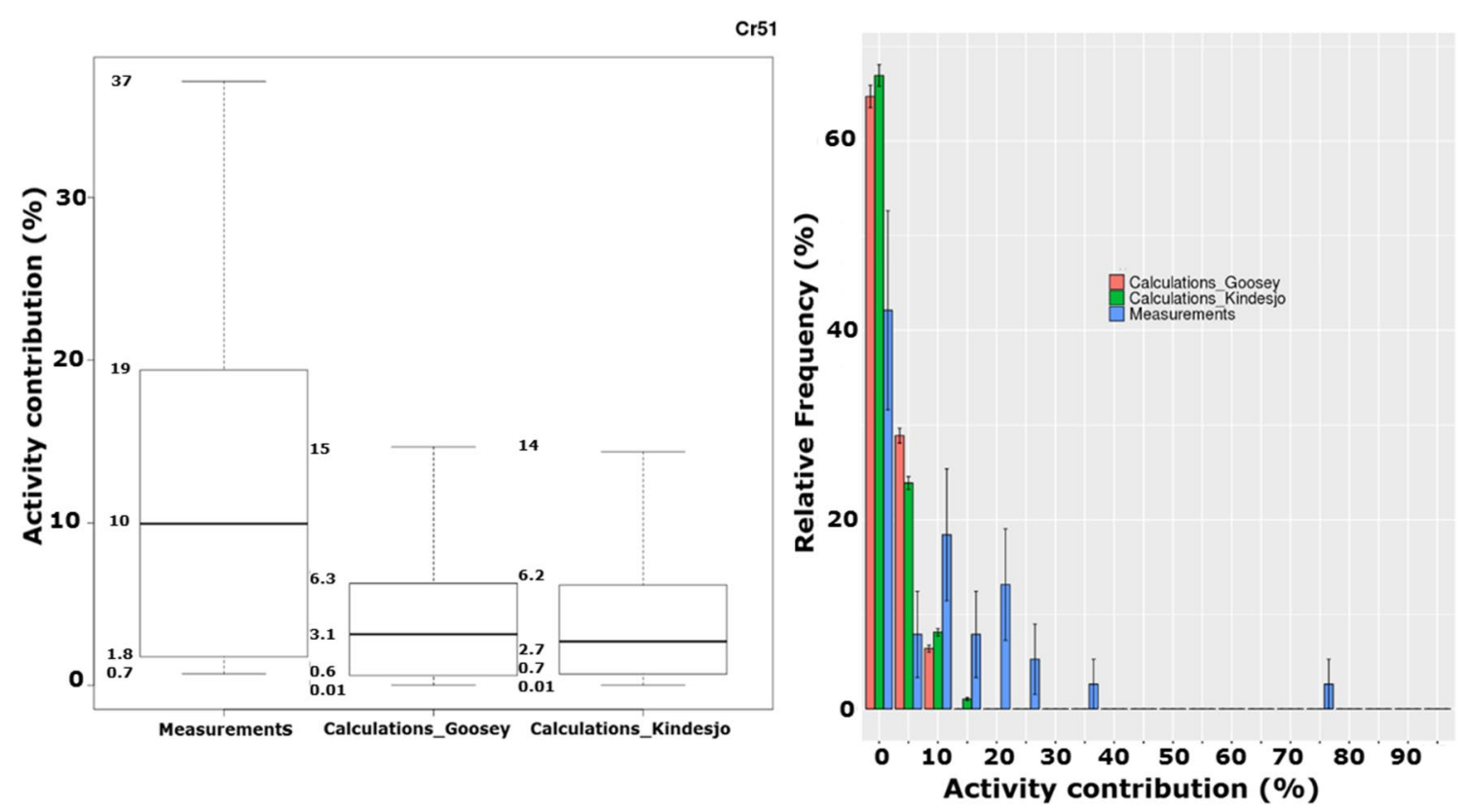

Fig. 11 Activity contribution comparison with measurements and ActiWiz calculations for Cr-51. The left figure represents a boxplot displaying first and third quartiles and the median (outliers excluded). The right figure is a frequency histogram of the activity contributions

Regarding the Be-7 radionuclide, the activity contribution behavior strongly diverges between the calculations and the measurements. One can easily notice in Fig. 8 that the measurement distribution reaches a maximum value of $100 \%$ of Be-7 while only $79 \%$ is found in the calculations. Moreover, the medians differ by a factor of 3 , higher in the case of the measurements. The relatively small Be-7 halflife (53.22 days) favors its disappearance in long cooling time scenarios for carbon-rich materials such as electronics. Within the sample population gamma-spectroscopy measurements are often carried out shortly after the irradiation $(>10 \mathrm{~h})$. This explains the reason behind the spread of the distribution from 0 to $100 \%$ (Fig. 8) in the case of the measurements. As mentioned previously this example highlights that a divergence of the distribution of irradiation/cooling scenarios between simulations and measurements becomes most notable for nuclides with short half-lives.

For Na-22 (see Fig. 9), contribution activity quartiles range from a factor 7 to 8 between measurements and ActiWiz calculations. It is crucial to note that the electronic equipment is often enclosed in an aluminum box (Fig. 1). This explains why Na-22 is identified with higher contribution activities in the measurements compared to the calculations since the aluminum boxes are not considered in the Goosey and Kindesjo material compositions.

Finally, for Co-58 and Cr-51, whose activity contributions are presented in Figs. 10 and 11, we observe a similar behavior like Be-7. Co-58 and $\mathrm{Cr}-51$ have short half-lives, 70.86 and 27.70 days respectively. As already mentioned, electronic components are often measured (with gamma spectroscopy) after short cooling times. This explains why they are identified with higher contribution activities in the measurements. Moreover, these radionuclides often have a rather low gamma spectroscopy MDA compared to others and hence increasing their activity contributions. However, we observe similarities in their probability distribution (histograms) even if the spread is higher in the measurements.

The ratios of various statistical quantities of the calculations and the measurements are shown in Table 3. One can see that for half of the radionuclides, the ratios range from 0.5 to 2 except for Na-22, Sn-113, Br-82, Sb-122/124, Cr-51, Co-58, Co-56 and Ag-110 m.

\section{Investigation of radionuclides appearing in the measurements and not in the calculations}

As shown in Fig. 2, some radionuclides are identified in gamma spectroscopy results and are absent in the ActiWiz calculations. These radionuclides are: Te-123m, Ta-182, $\mathrm{Rb}-83$, Se-75, Sn-113 and Y-88. The following sub-sections will address each of these nuclides.

\section{Te-123m production}

We note that Te- $123 \mathrm{~m}$ is identified in gamma spectroscopy results using the key gamma ray at $159 \mathrm{keV}$. However, Te$123 \mathrm{~m}$ presents an interference with Sc-47 at the $159 \mathrm{keV}$ gamma line. Since both nuclides are mono-energetic emitters 
Table 3 Ratio of statistical quantities between calculation and measurements for the 21 radionuclides of interest

\begin{tabular}{lcccccccccccc}
\hline Ratio calc/meas & Be-7 & Zn-65 & Mn-52 & Na-24 & Mn-54 & Na-22 & Co-60 & Sn-113 & V-48 & Sc-46 \\
\hline max & 0.79 & 0.78 & 0.57 & 2.09 & 0.91 & 0.25 & 0.54 & 0.07 & 0.61 & 1.65 \\
median & 0.29 & 1.06 & 0.06 & 0.61 & 1.51 & 0.17 & 0.46 & 0.02 & 0.76 & 0.44 \\
3rd quartile & 0.43 & 0.96 & 0.17 & 0.23 & 1.42 & 0.13 & 0.60 & 0.03 & 0.93 & 0.64 \\
\hline Ratio calc/meas & Sc-47 & Br-82 & Au-198 & Fe-59 & Sb-122 & Sb-124 & Cr-51 & Co-58 & Co-57 & Co-56 & Ag-110m \\
\hline max & 2.91 & 0.62 & 3.21 & 1.39 & 0.60 & 0.38 & 0.22 & 0.68 & 1.28 & 0.25 & 0.29 \\
median & 0.47 & 0.27 & 0.79 & 0.78 & 0.15 & 0.08 & 0.26 & 0.28 & 1.13 & 0.58 & 0.76 \\
3rd quartile & 1.04 & 0.30 & 2.13 & 0.74 & 0.34 & 0.14 & 0.31 & 0.32 & 1.27 & 0.88 & 0.86 \\
\hline
\end{tabular}

at $159 \mathrm{keV}$, it is not possible to accurately determine the nuclide emitter without external auxiliary information that is provided to the gamma spectroscopy algorithms.

Tellurium is occasionally used in the manufacturing process of electronics for thermoelectric and photoelectric devices. In particular, tellurium is used for the cooling of detectors, laser diodes, etc. Figure 12 shows that the crosssection of the reaction Te-122(n, $\gamma$ )Te-123m is high for lowenergy neutrons. Te-122 can be found with an abundance of $2.55 \%$ in natural tellurium. These data suggest that the gamma ray detected at $159 \mathrm{keV}$ came indeed from Te- $123 \mathrm{~m}$, and that tellurium should be added in the assumed material composition. Yet, the quantification of the amount requires a dedicated study and relatively large sample populations in order to obtain reliable estimates. Given that the radiotoxicity [9] of Te-123m is a factor of 10 smaller than Co-60 or Mn-54 and that the nuclide plays a minor role for historic waste due to its short half-life of 119 days, the overall impact of its negligence is deemed to be acceptable for the process of waste elimination. Yet, it clearly shows ways of possible improvements that might be interesting to be followed within a larger project aiming at improving calculation models.

\section{Ta-182 production}

Natural tantalum is composed mainly of Ta-181 (99.99\% in mass). For low energy neutrons, below $20 \mathrm{MeV}$, the production cross-sections of Ta-182 are plotted in Fig. 13. We can see that the Ta-182 production is driven by the radiative capture on Ta-181. From this consideration, we can conclude that the electronic equipment irradiated at CERN could include natural tantalum in the material compositions, and indeed tantalum is commonly used in electronic compounds for its electrical and thermal properties. However, in the calculations the electronic materials do not include any tantalum. As Ta-181 is found in the measurements, tantalum should be added to the reference chemical composition for improved accuracy. Yet, the quantification of the amount
Fig. 12 Low energy neutrons cross-sections for the production of Te-123m from natural tellurium elements (extracted from EXFOR/ENDF with EAF2010 library). For the reaction Te-122(n, $\gamma$ )Te-123, the multiplicity is on average 0.65 for the production of the ground-state and 0.35 for the production of the metastable

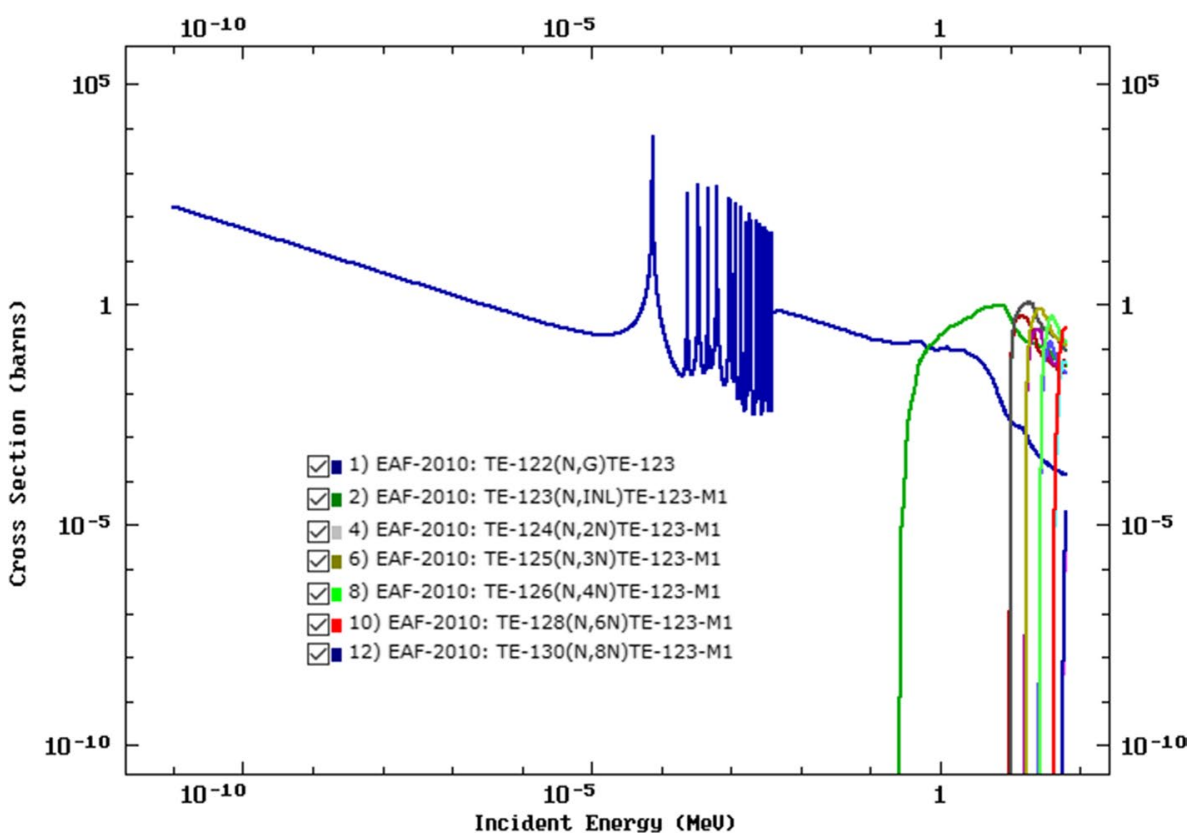


Fig. 13 Low energy neutrons cross-sections for the production of Ta-182 (extracted from EXFOR/ENDF with JEFF-3.3 library)

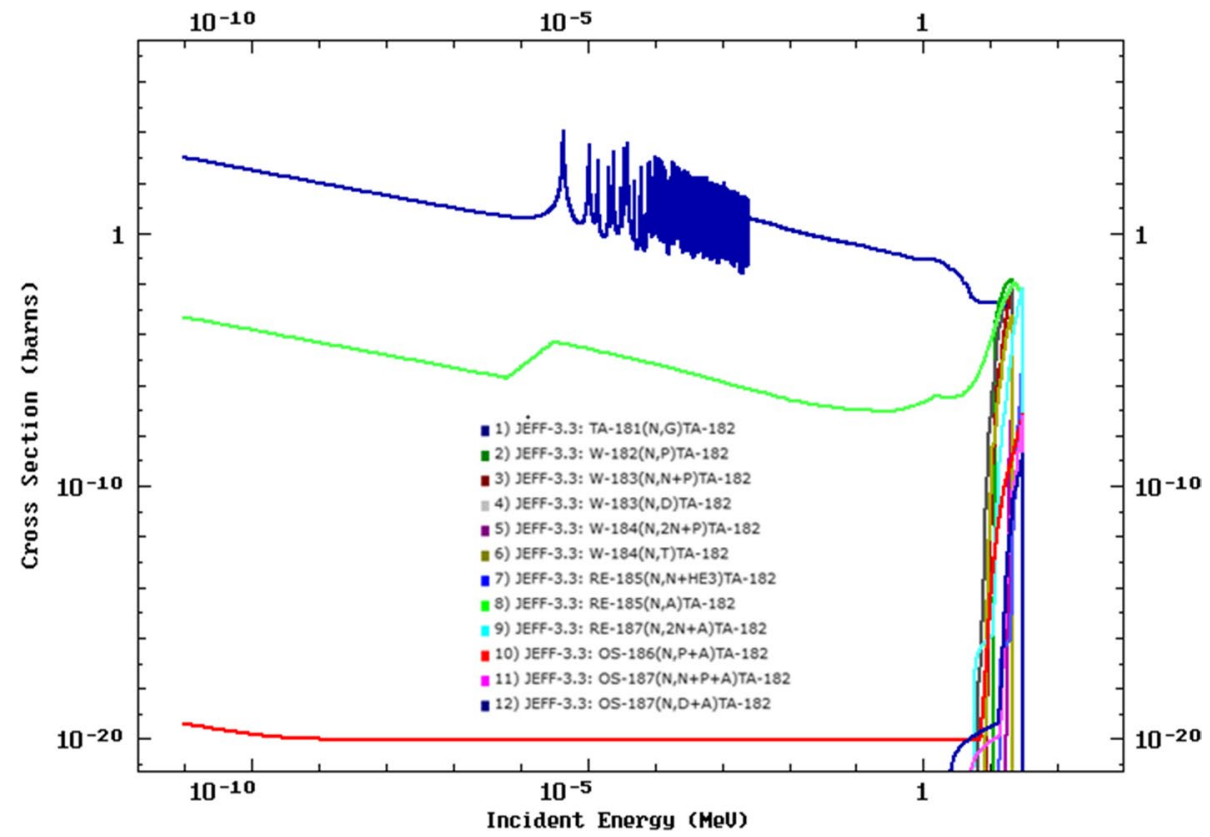

requires a dedicated study and relatively large sample populations in order to obtain reliable estimates.

Given that the radiotoxicity of Ta-182 is identical to the one of Co-60, the impact could be important on the characterization process. However, similarly to Te-123m also Ta-182 has a relatively short half-life of 114 days. Therefore, its importance for characterization of electronic waste with cooling periods longer than two years is certainly negligible, also considering that its abundance is likely to be well below $1 \%$ of the original amount. Yet, also this element could be included in further studies in order to complete the assumed chemical compositions.

\section{Se-75, Rb-83, Y-88 production}

These three radionuclides have short half-lives ( $<120$ days). They are consequently measurable only for short cooling times. The study presented in Refs. [10, 11] lists elements that can be identified in electronic circuits. In particular, selenium, zirconium, and strontium are identified with concentrations between 10 and $40 \mathrm{~g} \mathrm{ton}^{-1}$ based on a chemical composition study of electronic scraps [12].
In the present study, $\mathrm{Se}-75, \mathrm{Rb}-83$, and $\mathrm{Y}-88$ presented an activity contribution less than $1 \%$ in the ActiWiz calculations. They are found to contribute 5 times more to the percentage of total activity in gamma spectroscopy results than in the calculations. We note that their origin in the calculations comes from the elements lead and tin. However, this difference between the calculations and the measurements shows that other elements, not included in the material compositions could produce these radionuclides.

Table 4 qualitatively describes the main elements from which these radionuclides can be produced.

The elements shown in Table 4 can be present in electronic components with very low concentrations in the form of impurities.

In particular, the neutron production cross-section of Se-75 for natural selenium is very high ( 100 barns at low energy). Consequently, a tiny quantity of selenium in the material compound could lead to a non-negligible production of Se-75 after irradiation. However, the measured activity contributions of $\mathrm{Y}-88, \mathrm{Rb}-83$ and Se-75 is always, respectively, below $1 \%, 1 \%$, and $5 \%$ respectively. As the radiotoxicity of Y-88 is identical to the one of Co-60, those from $\mathrm{Rb}-83$ and $\mathrm{Se}-75$ are 10 times smaller, and these
Table 4 Production levels of $\mathrm{Y}-88, \mathrm{Rb}-83$ and $\mathrm{Se}-75$ in various irradiated materials

\begin{tabular}{lllllllll}
\hline & Molybdenum & Niobium & Rubidium & Ruthenium & Selenium & Strontium & Yttrium & Zirconium \\
\hline $\mathrm{Y}-88$ & $*$ & $* *$ & - & $*$ & - & - & $* * *$ & $* * *$ \\
$\mathrm{Rb}-83$ & $*$ & $*$ & $* * *$ & - & - & $* * *$ & $* *$ & $* *$ \\
$\mathrm{Se}-75$ & $*$ & $*$ & $*$ & - & $* * * *$ & $*$ & $*$ & - \\
\hline
\end{tabular}

A dash "-" represents no production and the higher the number of asterisks "*" the higher the element contribution to the radionuclide production 
radionuclides have short half-lives below 120 days, the overall impact of their negligence is deemed to be acceptable for the process of waste elimination having more than two years of cooling time. However, it clearly shows ways of possible improvements that might be interesting to be followed within a larger project aiming at improving calculation models.

\section{Sn-113 production}

Sn-113 originates from natural tin in the electronic components mainly via the reaction $\mathrm{Sn}-112(\mathrm{n}, \gamma) \mathrm{Sn}-113$. It has a frequency of occurrence of around $20 \%$ in the gamma spectroscopy results and $0 \%$ in the calculations (Fig. 2). We remind the reader that in this study, we only consider radionuclides which appear with more than $1 \%$ of contribution to the nuclide vector. However, the highest activity contribution of Sn-113 in the calculations is $0.36 \%$. Figure 14 below shows the histogram of the contribution of Sn-113 in the radionuclide vector. We see that in the gamma spectroscopy results, this contribution can reach 5\%. The ActiWiz calculations and gamma spectroscopy results are consistent for low activity contributions below $0.3 \%$ (Fig. 14). Hence, the absence of Sn-113 in the calculations (Fig. 2) is

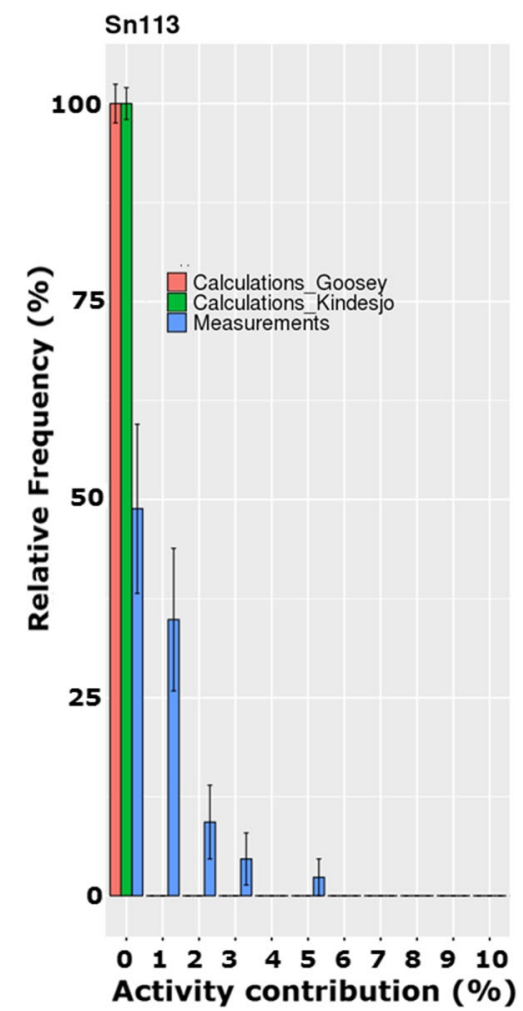

Fig. 14 Frequency histogram representing activity contribution comparison between measurements and ActiWiz calculations for Sn-113. Uncertainties are presented at 1 sigma a consequence of considering only radionuclides with more than $1 \%$ of activity contribution to the total activity.

\section{Conclusions}

The ActiWiz code is used with two different averaged material compositions for electronics to calculate nuclide inventories for a large range of typical exposure scenarios encountered at CERN.

Using a set of 218 gamma spectroscopy results performed on electronic equipment, we show that the activation calculations are statistically consistent with the measurement results in the sense that similar radionuclides are predicted to be of importance and as well as their contribution to the total activity in general matches within a factor of 2 . We find this level of agreement perfectly acceptable based on the more detailed analysis provided below.

For the main radionuclides (Mn-54, Co-57, Co-60, Zn-65, Ag-110 m), the calculations that are based on the Kindesjo and Goosey material compositions provide consistent activity contribution results with the gamma spectroscopy results. For radionuclides with short half-lives (Be-7, $\mathrm{Cr}-51$ and Co-58), discrepancies are observed, due to performing the measurements shortly after irradiation. The uniform distribution of scenarios described by a specific irradiation and cooling time will not necessarily represent the parameters of the measurements which show a certain, difficult to quantify, bias towards short cooling times. While the impact of this is minor for elements with long half-lives like Co-60 it understandably becomes more notable for elements with short half-lives like Be-7.

In the case of Na-22, the calculations show underestimation of the corresponding activity due to the presence of aluminum boxes surrounding electronic equipment in part of the analyzed samples.

A few radionuclides appeared in the measurement results but not in the calculations: Ta-182, Sn-113, Te-123m, Y-88 and Se-75. Our calculations and measurements do not allow us identifying with certainty the target element that produce Sn-113, Te-123m and Se-75. Regarding Ta-182 and Se-75, we know that the target elements are Ta and Se, but our data do not allow us to quantify their mass fraction. However, these radionuclides have short half-lives ( $<120$ days) and would not have effect on the radiotoxicity of radioactive wastes with cooling times above 2 years. If the activity evaluation of these radionuclides is required for some special application, we recommend relying on gamma spectrometry measurements rather than on analytical predictions - unless the complete and accurate chemical composition is available. Such composition could be identified by performing a more detailed study to quantify the amounts of elements that need to be added to the Kindesjo and Goosey material 
compositions. This could be achieved by performing calculations and measurements comparisons with controlled irradiation scenarios.

Also, some radionuclides appear in the calculations using Kindesjo's composition as well as in measurements, but they are missing in simulations based on Goosey's composition. These radionuclides are $\mathrm{Sc}-46 / 47$, coming from titanium, $\mathrm{Sb}-122 / 124$, coming from antimony and $\mathrm{Br}-82$, coming from bromine.

In conclusion this study shows that the average material compositions by Kindesjo [2] and Goosey [3], in combination with the generic radiation fields and the ActiWiz code allow for predicting radionuclide inventories which, in terms of their composition, are compliant with gamma spectroscopy measurement data over the last 14 years at CERN within a factor of two. Given the complexity of the material compound such agreement can be considered as highly satisfying. Finally, one can conclude that for accuracy driven studies, the most accurate composition would be an average of the Goosey's and Kindesjo elemental compositions. By using such a composition, the main radionuclides activities levels and occurrence frequencies would remain consistent between calculations and gamma spectroscopy measurements. Moreover, it would allow the Sc-46/47, $\mathrm{Sb}-122 / 124$ and Br-82 to appear in the calculations in quantities and frequencies similar to the ones from the gamma spectroscopy results. Additionally, these compositions are already the result of averages over measured sample populations. Considering the average composition of Goosey and Kindesjo would result in increasing the population they originate from. Hence the statistics would be more representative. However, for classification and penalizing studies (for radiotoxicity levels), the present study recommends the use of the Goosey elemental composition.

While small improvements to the respective material composition can be made, to account for radiologically less important isotopes that are identified during measurements, this result corroborates the overall applicability of the assumed generic chemical compositions as well as predictive power of the code for future studies involving electronics.

\section{Acknowledgements Open access funding provided by CERN (Euro-} pean Organization for Nuclear Research).

Open Access This article is licensed under a Creative Commons Attribution 4.0 International License, which permits use, sharing, adaptation, distribution and reproduction in any medium or format, as long as you give appropriate credit to the original author(s) and the source, provide a link to the Creative Commons licence, and indicate if changes were made. The images or other third party material in this article are included in the article's Creative Commons licence, unless indicated otherwise in a credit line to the material. If material is not included in the article's Creative Commons licence and your intended use is not permitted by statutory regulation or exceeds the permitted use, you will need to obtain permission directly from the copyright holder. To view a copy of this licence, visit http://creativecommons.org/licenses/by/4.0/.

\section{References}

1. Vincke H, Theis C (2017) ActiWiz 3-an overview of the latest developments and their application. J Phys Conf Ser 1046(1):012007. https://doi.org/10.1088/1742-6596/1046/1/01200 7

2. Kindesjo U (2002) An assessment of possible impacts of material substitution in electronic solders on the recycling of printed circuit boards. IIIEE master thesis at The International Institute for Industrial Environmental Economics, Lund, Sweden

3. Goosey M, Kellner R (2003) Recycling technologies for the treatment of end of life printed circuit boards. Circuit World Mag 29(3):33-37

4. Froeschl R, Theis C, Torre FL, Vincke H, Walter N (2012) Radiological hazard classification of material in CERN's accelerators. EDMS id 1184236 CERN-DGS-2012-003-RP-IR.I. Bergstrom. FLUKA estimated particle fluence in the four major LHC experiments for Actiwiz3. CERN EDMS 1723334

5. Venkataraman R, Bronson F, Atrashkevich V, Field M, Young BM (2003) Improved detector response characterization method in ISOCS and LabSOCS. In: Methods and Applications of Radioanalytical Chemistry (MARC VI) conference

6. Bronson F, Young BM, Atrashkevich V (1997) ISOCS mathematical calibration software for germanium gamma spectroscopy of small and large objects. Transactions of the American Nuclear Society Annual Meeting, June 1997

7. Menaa $\mathrm{N}$ et al (2011) Mathematical efficiency calibration with uncertain source geometries using smart optimization. In: 2011 2nd international conference on advancements in nuclear instrumentation, measurement methods and their applications

8. Frosio T, Menaa N, Duchemin C, Riggaz N, Theis C (2020) A new gamma spectroscopy methodology based on probabilistic uncertainty estimation and conservative approach. Appl Radiat Isot 155:108929

9. Bundesamt fuer Gesundheit (BAG/OFSP) (2018) Ordonnance sur la radioprotection ORAP

10. Zaffora B (2014) Radiological characterization of activated printed circuit boards for future elimination. Master thesis

11. Zaffora B, Magistris M (2016) Radiological characterization of activated printed circuit boards for future elimination. Appl Radiat Isot 113:40-46

12. Lehner T et al (1998) Integrated recycling of non-ferrous metals at boliden Ltd. Rönnskär Smelter. Current and future practices. The mineral, metals and material society. Sweden

Publisher's Note Springer Nature remains neutral with regard to jurisdictional claims in published maps and institutional affiliations. 